\title{
CHANDRA AND RXTE STUDIES OF THE X-RAY $/ \gamma$-RAY MILLISECOND PULSAR PSR J0218+4232
}

\author{
L. Kuiper ${ }^{1}$, W. Hermsen ${ }^{1}$, and B. Stappers ${ }^{2}$ \\ ${ }^{1}$ SRON National Institute for Space Research, Sorbonnelaan 2, 3584 CA Utrecht, The Netherlands \\ ${ }^{2}$ ASTRON, Postbus 2, 7990 AA Dwingeloo, The Netherlands
}

\begin{abstract}
We report on high-resolution spatial and timing observations of the millisecond pulsar PSR J0218+4232 performed with the Chandra X-ray Observatory (CXO) and the Rossi X-ray Timing Explorer (RXTE). With these observations we were able to study $a$ ) the possible spatial extent at X-ray energies of the DC source coincident with PSR $\mathrm{J} 0218+4232$ in detail $(\mathrm{CXO}), b$ ) the relative phasing between the X-ray, radio and $\gamma$-ray profiles (CXO and RXTE) and $c$ ) the spectral properties at energies beyond $10 \mathrm{keV}$ (RXTE). We found no indications for extended emission at $\mathrm{X}$-ray energies down to $\sim 1^{\prime \prime}$ scales and confirmed the presence of a point-like DC-component. The 2 non-thermal pulses in the X-ray profile are found to be aligned with 2 of the 3 pulses visible at radio-frequencies and more importantly with the two $\gamma$-ray pulses seen in the EGRET 100-1000 MeV pulse profile. The latter reduces now the random occurrence probability for the detected $\gamma$-ray signal to $\sim 10^{-6}$, which corresponds to a $4.9 \sigma$ detection significance. This strenghtens the credibility of our earlier claimed detection of pulsed high-energy $\gamma$-ray emission from this millisecond pulsar. The pulsed RXTE spectrum appears to extend to $\sim 20 \mathrm{keV}$ and can be described by a power-law with a photon index of 1.14, slightly softer than measured by BeppoSAX and XMM for energies below $10 \mathrm{keV}$. We compare the high-energy characteristics of PSR J0218+4232 with those of two other X-ray emitting millisecond pulsars PSR B1821-24 and PSR B1937+21.
\end{abstract}

\section{INTRODUCTION}

Millisecond pulsar PSR J0218+4232 is remarkable in many respects. It has a very broad and complex radio pulse profile with 3 pulses (Navarro et al. 1995, Stairs et al. 1999), and a 13 $\pm 9 \%$ radio DC contribution (Kuiper et al. 2002), suggesting that we continously look into the radio beam. At X-ray energies a double peaked profile has been observed (ROSAT HRI: Kuiper et al. 1998; BeppoSAX MECS: Mineo et al. 2000; Chandra HRC-S: Kuiper et al. 2002), and also in this regime a DC-component has been detected, significantly only at soft X-rays, and possibly originating from a spatially extended region (Kuiper et al. 1998). The pulsed 0.1-10 keV X-ray spectrum turns out to be very hard with a photon index in the range $0.5-1.0$. Surprisingly, marginally significant pulsations have also been reported at high-energy ( $>100 \mathrm{MeV}$ ) $\gamma$-rays from a sky region containing an $\sim 11 \sigma$ EGRET source, 3EG J0222+4253, which is positionally consistent with PSR J0218+4232 (Kuiper et al. 2000). The 100-1000 MeV $\gamma$-ray profile shows one prominent pulse and a weaker broader one, coincident with 2 of the 3 radio-pulses. The high-energy $\gamma$-ray emission is consistent with being $100 \%$ pulsed and its spectrum can be described by a power-law with a photon index $\sim 2.6$. Unfortunately, inaccuracies in the absolute timing of the events during the ROSAT and BeppoSAX observations made direct comparisons of the soft and medium energy X-ray profiles with the radio and high-energy $\gamma$-ray profile impossible. The aim of the Chandra and RXTE observations was to shed light on the spatial extent of the X-ray counterpart of PSR J0218+4232 (CXO), to obtain high-resolution X-ray pulse profiles which could be phase related to the radio and $\gamma$-ray profiles (CXO and RXTE), and to extend the spectral coverage to energies beyond $10 \mathrm{keV}$ (RXTE). 


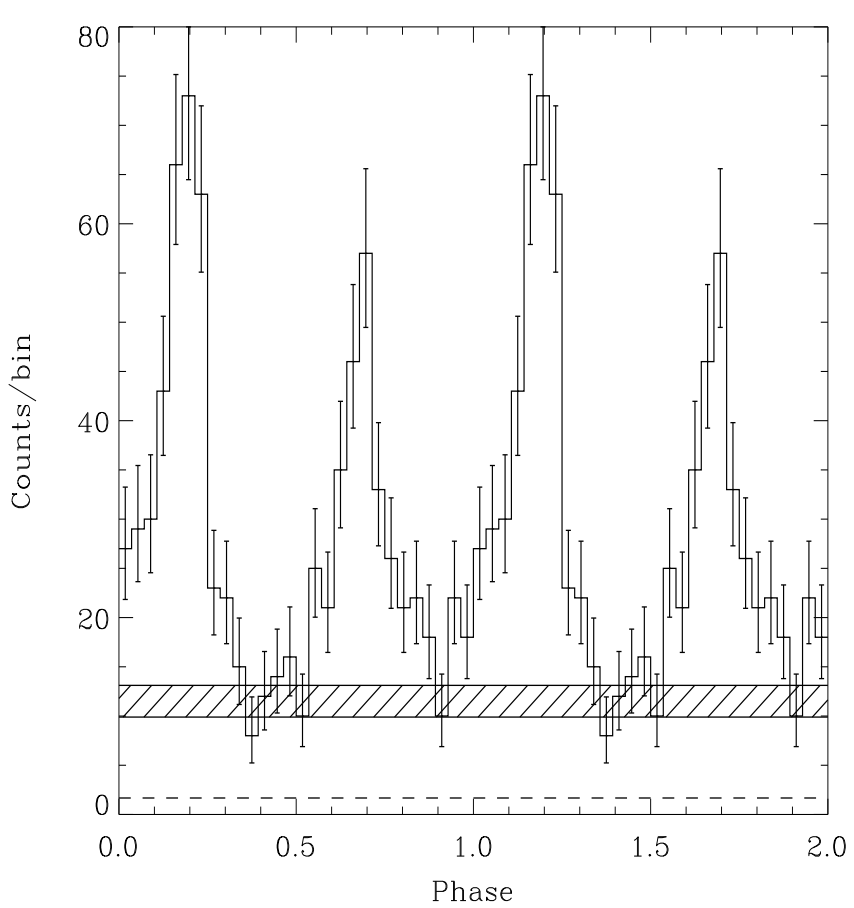

Fig. 1. Pulse profile of PSR J0218+4232 (0.08-10 $\mathrm{keV}$ ) as observed by Chandra HRC-S in imaging mode. Two cycles are shown for clarity. Typical error bars are indicated. The background level from the spatial analysis is shown as a dashed line, while the hatched area indicates the DC-level $\pm 1 \sigma$. The DC-fraction is $0.36 \pm 0.06$. (see also Kuiper et al. 2002)

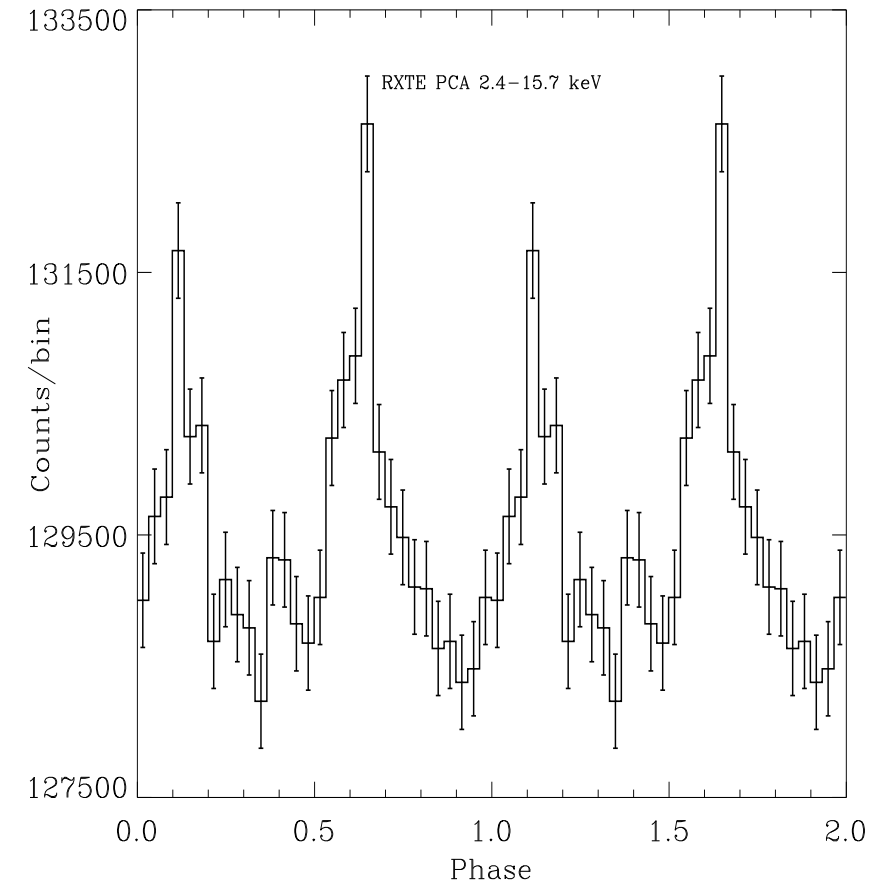

Fig. 2. Pulse profile of PSR J0218+4232 as observed by the RXTE PCA in the $2-16 \mathrm{keV}$ energy window (30 bins; only data from the PCA top layer are used). Two cycles are shown for clarity. Error bars indicated are $1 \sigma$. The modulation significance is $\sim 12.5 \sigma$ applying a $Z_{4}^{2}$ test. Notice the morphology change relative to the soft X-ray profile for the Chandra HRC-S shown in Figure 1

\section{CHANDRA AND RXTE RESULTS}

\section{CHANDRA Observations and Results}

Chandra observed PSR J0218+4232 twice with the High Resolution Cameras HRC-I and HRC-S. These cameras are multichannel plate detectors sensitive to X-rays in the $0.08-10 \mathrm{keV}$ range with no spectral resolution. The first observation on 22-12-1999 with the HRC-I in the focal plane of the telescope mirror and the second observation on 5-10-2000 with the HRC-S in the special imaging mode yielded effective exposures of $74.11 \mathrm{ks}$ and $73.21 \mathrm{ks}$, respectively. PSR J0218+4232 was detected significantly $(\sim 85 \sigma)$ in the first observation. Its spatial extent is consistent with that of a point-source. Thus we have no evidence of extended X-ray emission from PSR J0218+4232 at scales larger than $\sim 1^{\prime \prime}$.

The second HRC-S observation was devoted to the timing analysis of PSR J0218+4232. Phase folding the barycentered times of the X-ray events falling within 1". 5 from the centroid of the X-ray counterpart of PSR J0218+4232 yielded the well-known double peaked profile with high statistics (see Figure 1). The deviation from being flat applying a $Z_{6}^{2}$-test is $15.2 \sigma$. Indicated in Figure 1 as a hatched area is the DC or unpulsed level $( \pm 1 \sigma)$ determined using a bootstrap method (Swanepoel et al. 1996). Also indicated as a dashed line is the background level derived from an imaging study. The pointlike DC component can clearly be discerned in Figure 1 The fraction of source counts over the entire 0.08-10 keV energy range from this DC-component is $0.36 \pm 0.06$. Combining the Chandra HRC-S DC count rate with those observed by the ROSAT HRI and BeppoSAX MECS and assuming a spectral model for the DC emission we can put constraints on the spectral parameters. For a power-law model the $1 \sigma$-range for the photon index is 1.3-1.85, much softer than the pulsed emission (Kuiper et al. 2002).

Chandra's absolute timing accuracy is within $200 \mu$ s (Tennant et al. 2001) allowing detailed phase comparisons with the radio and $\gamma$-ray profiles. It turns out that the two non-thermal X-ray pulses are aligned with the two $\gamma$ ray pulses and two of the three radio pulses (see also the section about the Rossi X-ray Timing Explorer (RXTE) 
PCA/HEXTE timing analyses and Figure 4) given the timing inaccuracies of the instruments. The random occurrence probability that we are dealing with a $100-1000 \mathrm{MeV} \gamma$-ray profile with a $3.5 \sigma$ modulation significance and alignment between the X-ray and $\gamma$-ray pulses decreases to about $10^{-6}$ i.e. the significance of the detection of the pulsed $\gamma$-ray signal from PSR J0218+4232 improves to $\sim 4.9 \sigma$. For more details on the Chandra results, see Kuiper et al. (2002).

\section{RXTE Observation and Results}

RXTE observed PSR J0218+4232 for about $200 \mathrm{ks}$ between 26-12-2001 and 7-01-2002 (MJD time span 52269 52281). The Proportional Counter Array PCA (2-60 keV) data were obtained in the Good Xenon mode, time tagging each event with a $0.9 \mu$ s time resolution. The two detector clusters of the High Energy X-ray Timing Experiment HEXTE (10-250 keV) operated in staring mode, and the science mode was E_8us_256_DX1F allowing spectroscopic studies with 256 channels and a time tag resolution of $7.6 \mu$ s. During the observation period PSR J0218+4232 was regularly monitored by the Dutch pulsar machine PuMa (Voûte et al.2002) installed at the Westerbork Synthesis Radio Telescope (WSRT) allowing high precision timing studies.

\section{RXTE PCA/HEXTE Timing Analyses}

In the timing analysis of the PCA data the barycentered event times were folded with the newly obtained WSRT PuMa ephemeris (MJD range: 51386 - 52278) as well as with an earlier ephemeris based on timing measurements at Jodrell Bank (MJD range: 49092 - 51462). No significant differences in the X-ray pulse morphologies were seen folding the events with the different ephemerides.

The modulation significance in the $2.4-15.7 \mathrm{keV}$ window is $12.5 \sigma$ applying a $Z_{4}^{2}$-test: a 30 bin pulse profile is shown in Figure 2 The two X-ray pulses are located at approximately the same phases as in the CXO HRC-S lightcurve, however, the relative strengths of the pulses have changed. The pulsed signal can be detected up to $\sim 20$ $\mathrm{keV}$. The significances in the 2.4-7.8 keV and 7.8-15.7 keV windows are 10.6 and $6.0 \sigma$, respectively, applying again a $Z_{4}^{2}$-test. Therefore, for the first time significant pulsed emission has been detected at energies beyond $\sim 8 \mathrm{keV}$.

The HEXTE data were also screened using timeline filters. Pulse-phase folding the event times from the combined Cluster A + B screened datasets yielded pulse-phase distributions in 256 PHA channels. The pulsed signal has been detected in the lower PHA channels $9-16$, corresponding to $10.3-17.5 \mathrm{keV}$, reaching a detection significance of $\sim 3.7 \sigma$. A compilation of PCA/HEXTE pulse profiles in three different energy windows is shown in Figure 3 .

It is now also possible to cross-correlate the Chandra and RXTE profiles in the overlapping energy range. Taking into account the internal delays (PCA $16 \mu \mathrm{s}$; Chandra HRC-S 19.5 $\mu \mathrm{s}$ ) we found that the Chandra clock runs $105 \pm 17 \mu \mathrm{s}$ ahead the RXTE clock. This is a significant improvement upon a previous estimate based on a Chandra-RXTE correlation study of the much slower rotating Crab pulsar, for which absolute timing analysis is further complicated because of irregular Dispersion Measure variations. The Chandra phases have therefore been adjusted to the RXTE profile by applying a back-shift of 0.045 . Figure 4 finally, shows the comparison in absolute phase of the PSR J0218+4232 Chandra HRC-S and RXTE PCA X-ray profiles with the radio and $\gamma$-ray profiles. The non-thermal $\mathrm{X}$-ray pulses appear to be aligned within the timing uncertainties with two of the three radio pulses and with the two $\gamma$-ray pulses.

RXTE PCA/HEXTE Spectral Analyses

In the spectral analysis of the PCA data we treated the 5 Proportional Counter Units (PCU's) constituting the PCA separately. Effective exposure times for the individual PCU's are determined excluding time periods during which an increased background is encountered e.g. during a South Atlantic Anomaly passage. Moreover, time intervals are ignored when the angle between target and Earth Horizon is less than $5^{\circ}$ or the target aspect angle is larger than 0.05 . We further considered only event triggers from the top Xenon layer. Response matrices and sensitive areas were derived for each PCU separately using the ftools (v5.2, release 18-6-2002) program pcarsp (v8.0) and xtefilt (v1.7).

Next, for each individual PCU pulse-phase histograms have been generated from the screened PCU event datasets in 256 Pulse Height Analyzer (PHA) channels. In order to determine the pulsed counts from PSR J0218+4232 in different PHA slices we first made a profile template based on a fit of the total 2-16 keV pulse profile in terms of two asymmetric Lorentzians atop a flat background. The two asymmetric Lorentzians (fixed shape and position) were fitted, each with free normalization, atop a flat background with a free scale to the measured pulse-phase distribution for each selected PHA interval. The sum of the two normalizations constitutes then the total number of pulsed counts per PHA interval.

A simple absorbed power-law was fitted to the data using a fixed $\mathrm{N}_{\mathrm{H}}$ of $5 \cdot 10^{20} \mathrm{~cm}^{-2}$ to the combined PCU pulsed 


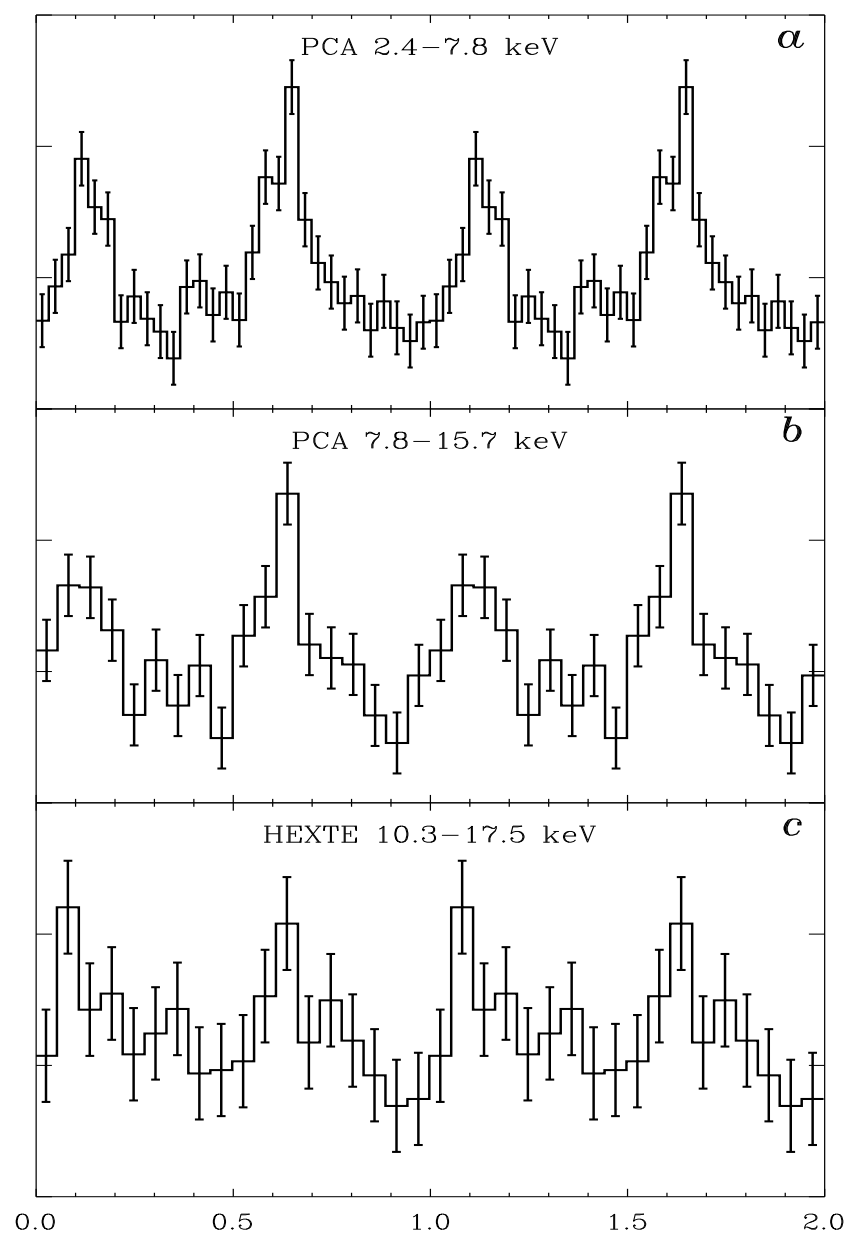

Phase

Fig. 3. Pulse profiles of PSR J0218+4232 as measured by the RXTE PCA for the 2.4-7.8 and 7.8-15.7 keV energy windows and RXTE HEXTE for the 10.3$17.5 \mathrm{keV}$ energy window.

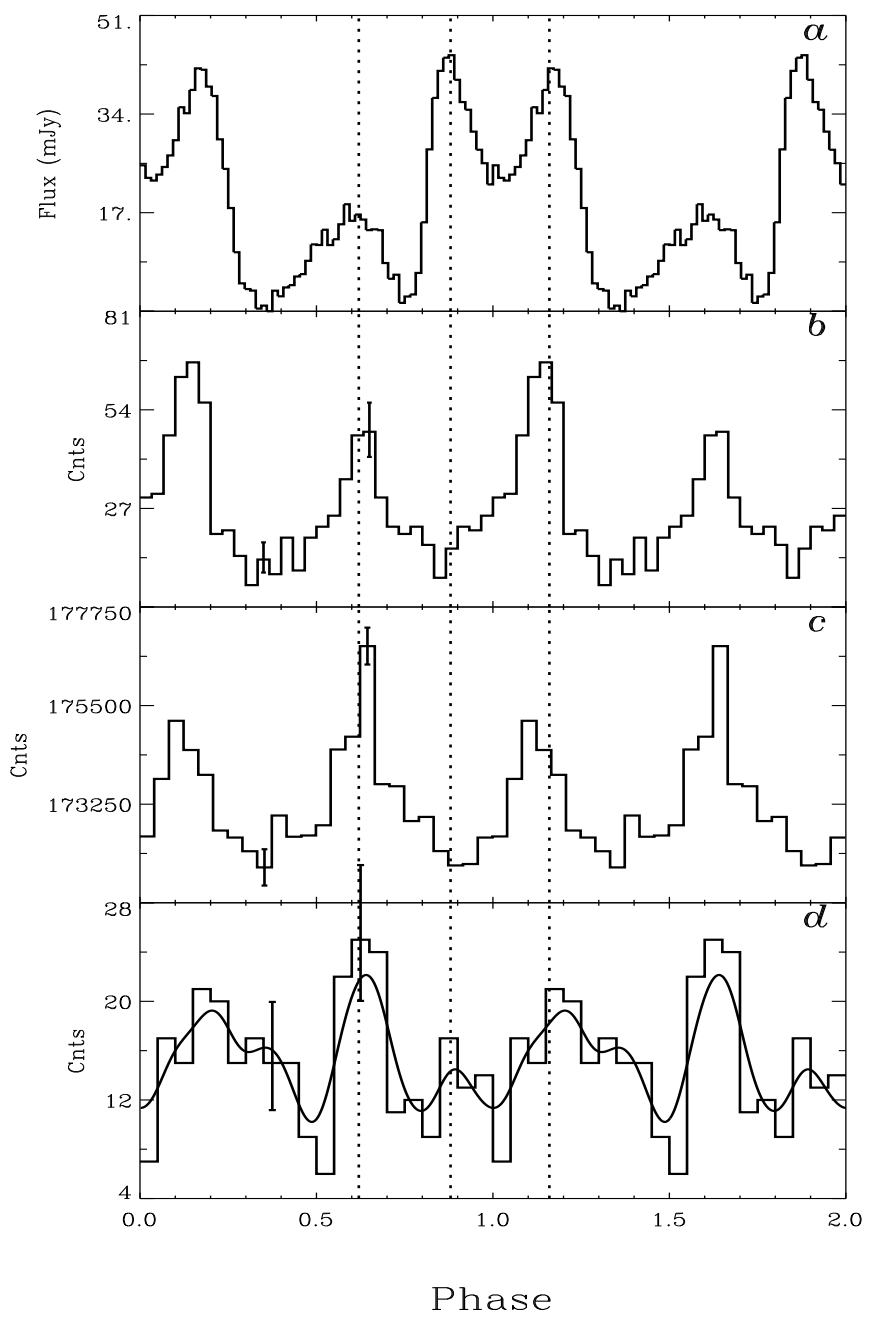

Fig. 4. Multi-wavelengths pulse profiles of PSR J0218+4232 in absolute phase. From top to bottom: Radio: $610 \mathrm{MHz}, \mathrm{X}$-rays: Chandra HRC-S 0.08-10 keV and RXTE PCA 2-16 keV, and $\gamma$-rays: CGRO EGRET $0.1-1 \mathrm{GeV}$.

counts, taking into account the individual PCU responses and screened exposures. The best fit total pulsed spectrum has a photon index of $1.14 \pm_{0.04}^{0.03}$ over the full PCA energy range. Using the best fit PCA based model templates for the two peaks we also revisited the BeppoSAX total pulsed emission by applying similar fit procedures in different BeppoSAX MECS energy intervals. We obtained for the MECS energy range a slightly harder photon index of $0.78 \pm_{0.10}^{0.08}$, which is a bit different, but consistent with the earlier reported value of 0.61 (Mineo et al. 2000). A joint fit of the revisited BeppoSAX MECS and RXTE PCA spectral measurements yielded a photon-index of $0.99 \pm 0.03$ over the entire MECS/PCA energy range. Similar fit techniques as used for the PCA have been employed to determine the number of pulsed counts for selected HEXTE PHA intervals. The effective HEXTE Cluster A and B exposures have been determined using ftools program hxtdead (v2.0.0). The actual exposures are typically reduced by $30 \%$ due to deadtime effects. Response matrices and sensitive areas have been derived using ftools program hxtarf (v1.9) taking into account aspect offsets between target and pointing. The HEXTE total pulsed counts for given PHA slices are not fitted through forward folding assuming a spectral model in view of the limited source count statistics, but instead the count rates are converted to flux values by estimating the effective sensitive area in a certain PHA slice assuming a power-law input photon spectrum with index 1. This approach yielded one significant flux point for the 10.3-17.5 $\mathrm{keV}$ energy window and upper-limits beyond this range. The newly derived RXTE PCA \& HEXTE and revisited BeppoSAX MECS flux measurements are shown in Figure 5 along with the ROSAT HRI (0.1-2.4 keV) flux point and 


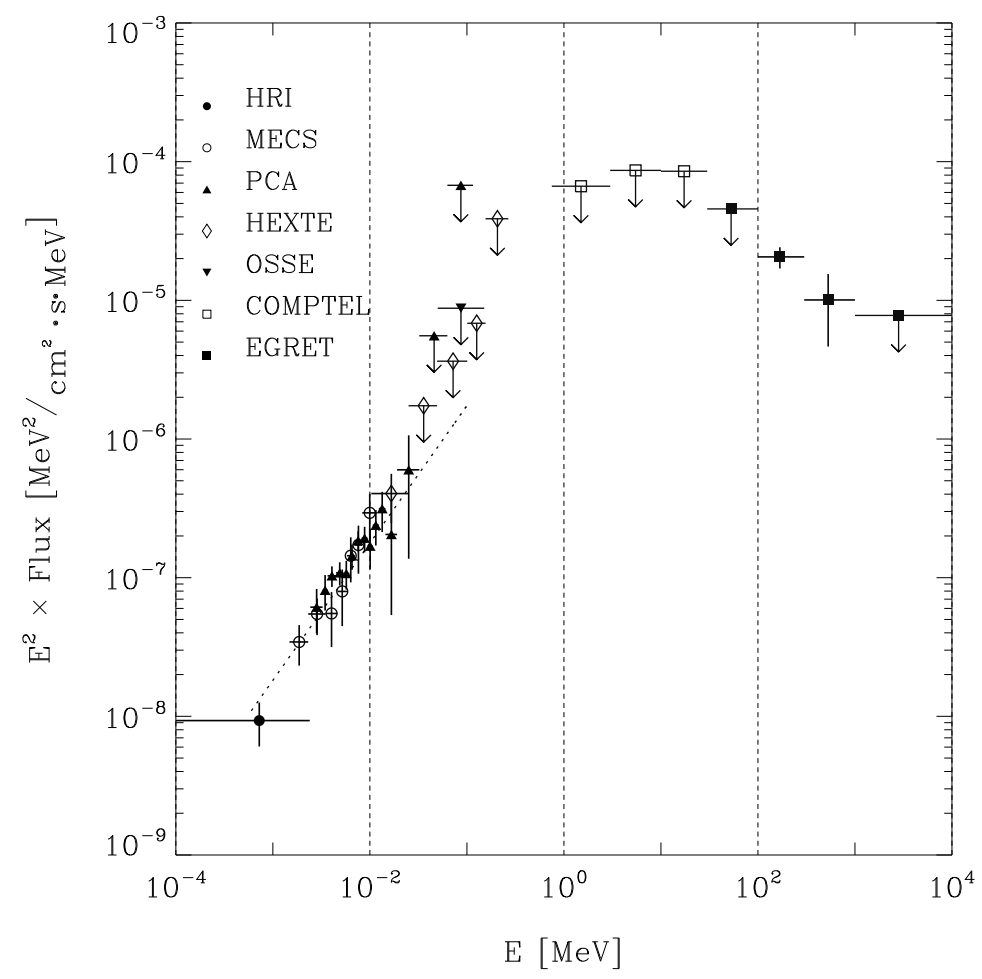

Fig. 5. Pulsed high-energy spectrum of PSR J0218+4232 in a $v \times F_{v}$ representation from soft X-rays up to high-energy $\gamma$-rays including the newly derived RXTE PCA/HEXTE and revisited BeppoSAX flux measurements and a spectral fit (dotted line; extrapolated up to $100 \mathrm{keV}$ ) based on XMM EPIC-PN total pulsed data (fit range: 0.6-10 keV; Webb, 2003).

CGRO OSSE, COMPTEL and EGRET flux values and upper limits (see Kuiper et al. 2000). The PCA flux points are multiplied by 0.81 , because the normalization of the PCA spectra turns out to be too high by 20\% based on BeppoSAX and RXTE PCA cross-calibrations (this work using Crab RXTE PCA data; see also Kuulkers et al. 2003). Superposed as dotted line is also a recent estimate of the pulsed spectrum of PSR J0218+4232 using XMM EPIC-PN data (Webb, 2003), which nicely overlaps with current flux measurements.

\section{CONCLUSION AND DISCUSSION}

From recently performed Chandra and RXTE observations of PSR J0218+4232 we learned that: a) the two non-thermal X-ray pulses are aligned with the $\gamma$-ray pulses and with two of the three radio pulses, b) pulsed emission has been detected significantly up to $\sim 20 \mathrm{keV}$, and c) the X-ray DC component is point-like down to $\sim 1^{\prime \prime}$ scales with a (much) softer spectrum than the pulsed emission.

How unique is PSR J0218+4232? So far, in X-rays three radio ms-pulsars have been detected showing hard $\mathrm{X}$-ray spectra with narrow pulses and having high X-ray luminosities. A comparison in absolute phase of the X-ray and radio pulse profiles of this small sample, comprised of PSR J0218+4232, PSR B1821-24 and PSR B1937+21, is shown in Figure 6 Some of the X-ray pulses are clearly aligned with radio pulses, while others are only approximately. The geometries i.e. the magnetic inclinations and observer/spin-axis angles are very different for these pulsars, implying that we can not draw simple conclusions from the phase comparisons and that 3D modeling of the magnetospheric electro-magnetic output is required using the exact geometry to explain the high-energy and radio profiles. It can be noted that these three millisecond pulsars are among the top four ranking millisecond pulsars according to their total spin-down luminosity.

In the spectral domain the high-energy pulsed spectra of PSR B1821-24 and PSR B1937+21 could be very similar to that of PSR J0218+4232. For PSR B1821-24 we found using archival RXTE PCA/HEXTE and ASCA GIS data that the total pulsed spectrum can be described by a power-law with photon index of $1.15 \pm 0.02$ for the PCA $(\sim 2-20 \mathrm{keV})$ and $1.30 \pm 0.07$ for the GIS $(\sim 0.8-10 \mathrm{keV})$. A joint fit of the ASCA GIS and RXTE PCA data (normalization correction of 0.81 applied) yields a photon-index of $1.13 \pm 0.02$ assuming an absorbing Hydrogen 


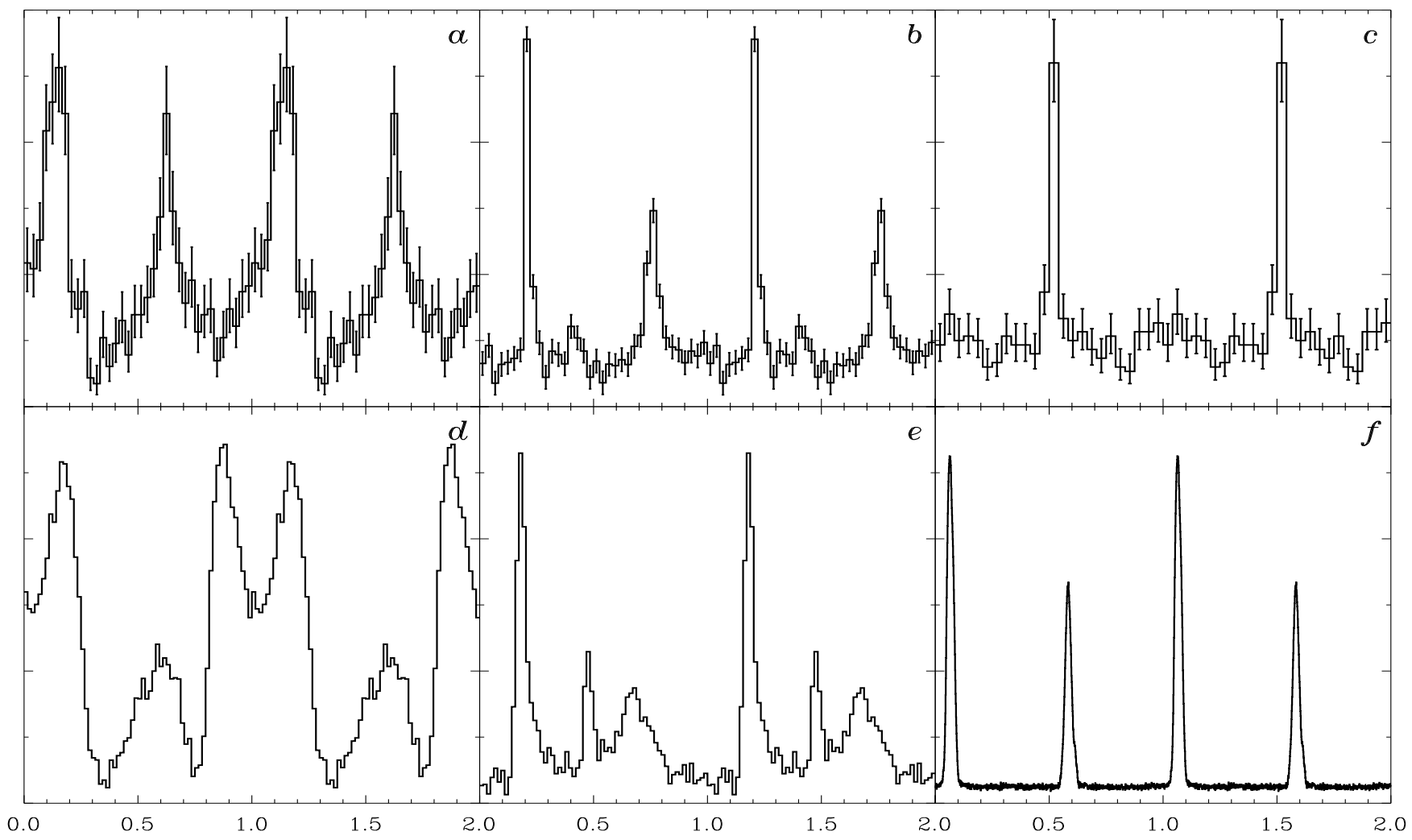

Phase

Fig. 6. Comparison in absolute phase of the three high-luminosity and hard X-ray millisecond pulsars PSR J0218+4232 (left; this work), PSR B1821-24 (middle; reanalysis of RXTE data) and PSR B1937+21 (right; alignment according to Takahashi et al. 2001) at X-rays (top panels) and radio-frequencies (bottom panels).

column of $1.6 \cdot 10^{21} \mathrm{~cm}^{-2}$. The total (i.e. pulsed + DC) spectrum of PSR B1821-24 derived recently using CXO ACIS data can be described by a power-law with photon index $1.20 \pm_{0.13}^{0.15}$ (Becker et al. 2003). Both the photon index and normalization of this total spectrum are consistent with the numbers we determined for the pulsed component alone, leaving little room for a DC-component. At high-energy $\gamma$-rays upper-limits are reported (Fierro et al. 1995). The pulsed high-energy spectrum of PSR B1821-24 from soft X-rays up to high-energy $\gamma$-rays is shown in Figure 7 The genuine spectrum could be very similar in shape as shown for PSR J0218+4232 in Figure 5

For PSR B1937+21 pulsed X-ray emission was detected by Takahashi et al. (2001). Nicastro et al. (2002) measured a photon index of $1.71 \pm 0.07$ for the total spectrum for energies up to $10 \mathrm{keV}$, but, sofar, at higher X-ray and gamma-ray energies no detections have been reported. The fact that PSR J0218+4232 is at present the only millisecond pulsar detected at gamma-ray energies can be merely due to the "modest" sensitivity of previous highenergy instruments for energies above e.g. $30 \mathrm{keV}$. Another reason might be that the present catalog of millisecond radio pulsars is still very incomplete. Namely, part of the unidentified high-energy gamma-ray sources in the COS-B (Swanenburg et al. 1981) and EGRET (Hartman et al. 1999) catalogues might have as counterparts millisecond pulsars which have been missed sofar in radio searches. The high-energy spectrum measured for PSR J0218+4232 (hard $\mathrm{X}$-ray and soft high-energy gamma-ray spectrum) is indeed reminiscent of that inferred for part of these unidentified sources and there are not many other candidate counterparts for the stable unidentified gamma-ray sources, excluding millisecond pulsars and normal radio pulsars.

Therefore: 1) Millisecond pulsars with hard X-ray spectra are good candidates for detection with future gammaray telescopes (e.g. with the recently launched ESA satellite INTEGRAL, sensitive between $15 \mathrm{keV}$ and $5 \mathrm{MeV}$, and the future Italian mission AGILE and NASA's GLAST, both sensitive to gamma rays with energies above about 30 $\mathrm{MeV})$;2) Accurate source localization of unidentified gamma-ray sources with future gamma-ray missions might lead 


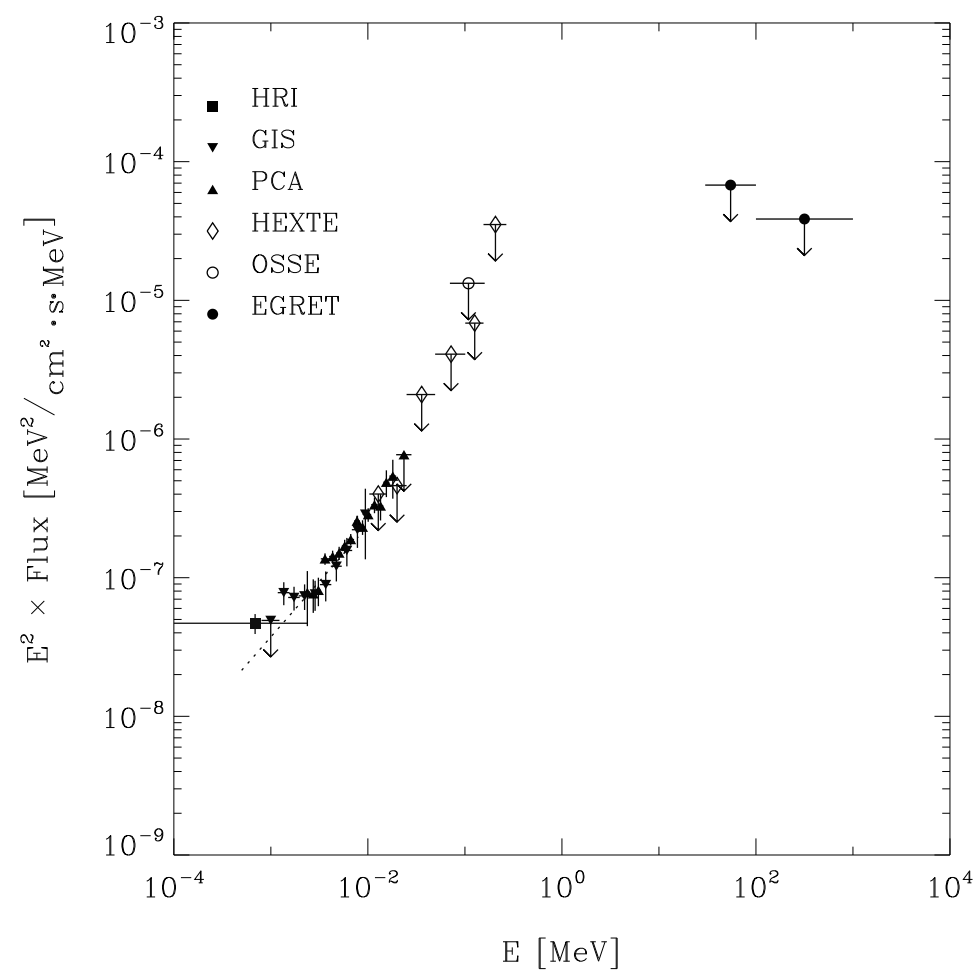

Fig. 7. Total pulsed high-energy spectrum of PSR B1821-24 in a $v \times F_{v}$ representation from soft X-rays up to high-energy $\gamma$-rays. The ROSAT HRI, ASCA GIS, RXTE PCA and RXTE HEXTE flux values/upper limits are determined in this work. The dotted line shows the total spectrum (0.5 - $8 \mathrm{keV})$ of PSR B1821-24 as derived from CXO ACIS data (Becker et al. 2003). EGRET upper limits are from Fierro et al. (1995).

to detections of new millisecond pulsars at radio wavelengths, followed by the identification of the pulsed signals in the X-ray and gamma-ray data.

We expect that PSR J0218+4232 will not remain for long the only millisecond pulsar detected at gamma-ray energies.

\section{References}

Becker, W., D. A. Swartz, G. G. Pavlov, et al., Chandra X-ray observatory observations of globular cluster M28 and its millisecond pulsar PSR B1821-24, Astrophys. J. (in press), 2003.

Fierro, J., Z. Arzoumanian, M. Bailes, et al., EGRET High-Energy gamma -Ray Pulsar Studies. II. Individual Millisecond Pulsars, Astrophys. J., 447, 807-812, 1995.

Hartman, R. C., D. L. Bertsch, S. D. Bloom, et al., The Third EGRET Catalog of High-Energy Gamma-Ray Sources, Astrophys. J. Supp., 123, 79-202, 1999.

Kuiper, L., W. Hermsen, F. Verbunt, et al., Detection of pulsed X-ray emission from the binary millisecond pulsar PSR J0218+4232, Astron. Astrophys., 336, 545-552, 1998.

Kuiper, L., W. Hermsen, F. Verbunt, et al., The likely detection of pulsed high-energy gamma -ray emission from millisecond pulsar PSR J0218+4232, Astron. Astrophys., 359, 615-626, 2000.

Kuiper, L., W. Hermsen, F. Verbunt, et al., High-Resolution Spatial and Timing Observations of Millisecond Pulsar PSR J0218+4232 with Chandra, Astrophys. J., 577, 917-922, 2002.

Kuulkers, E., P. R. den Hartog, J.J.M. in 't Zand, et al., Photospheric radius expansion X-ray bursts as standard candles, Astron. Astrophys., 399, 663-680, 2003. 
Mineo, T., G. Cusumano, L. Kuiper, et al., The pulse shape and spectrum of the millisecond pulsar PSR J0218+4232 in the energy band 1-10 keV observed with BeppoS AX, Astron. Astrophys., 355, 1053-1059, 2000.

Navarro, J., A. G. de Bruyn, D. A. Frail, et al., A Very Luminous Binary Millisecond Pulsar, Astrophys. J., 455, L55-L58, 1995.

Nicastro, L., G. Cusumano, L. Kuiper, et al., The 2-10 keV emission properties of PSR B1937+21, in proceedings of 270. WE-Heraus Seminar on Neutron Stars, Pulsars and Supernova Remnants, MPE-Report 278, pages 87-90, 2002.

Stairs, I. H., S. E. Thorsett, F. Camilo, et al., Coherently Dedispersed Polarimetry of Millisecond Pulsars, Astrophys. J. Supp., 123, 627-638, 1999.

Swanenburg, B. N., K. Bennett, G. Bignami, et al., Second COS B catalog of high-energy gamma-ray sources, Astrophys. J., 243, L69-L73, 1981.

Swanepoel, J. W. H., C. F. de Beer, and H. Loots, Estimation of the Strength of a Periodic Signal from Photon Arrival Times, Astrophys. J., 467, 261-264, 1996.

Takahashi, M., S. Shibata, K. Torii, et al., Pulsed X-Ray Emission from the Fastest Millisecond Pulsar: PSR B1937+21 with ASCA, Astrophys. J., 554, 316-321, 2001.

Tennant, A. F., W. Becker, M. Juda, et al., Discovery of X-Ray Emission from the Crab Pulsar at Pulse Minimum, Astrophys. J., 554, L173-L176, 2001.

Voûte, J. L. L., M. L. A. Kouwenhoven, P. C. van Haren, et al., PuMa, a digital Pulsar Machine, Astron. Astrophys., 385, 733-742, 2002.

Webb, N. A., Private communication, 2003. 